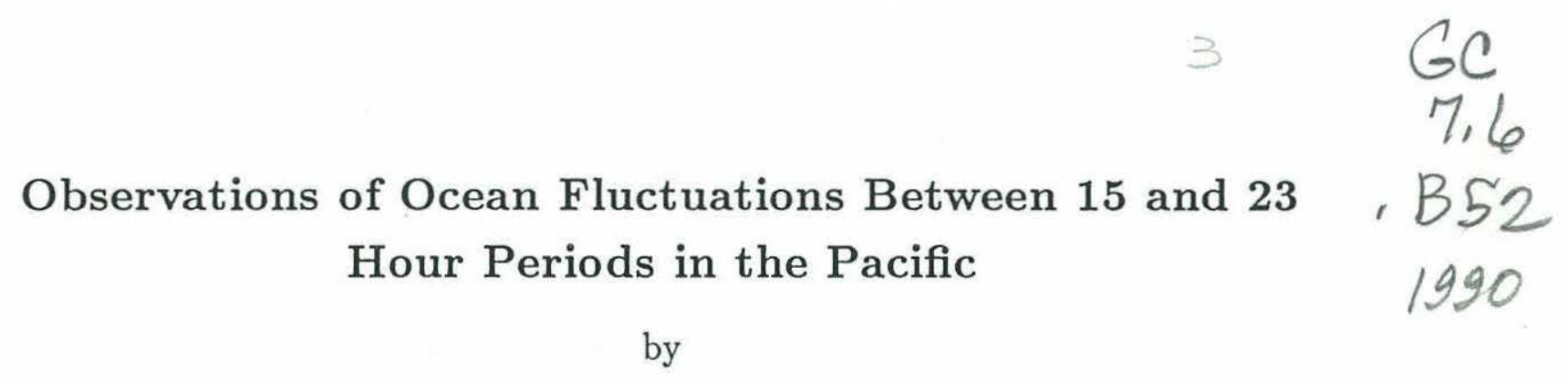

\author{
Wayne Richard Blanding \\ B.S., Systems Engineering, U.S. Naval Academy (1982) \\ Submitted in partial fulfillment of the \\ requirements for the degree of \\ OCEAN ENGINEER \\ at the \\ MASSACHUSETTS INSTITUTE OF TECHNOLOGY \\ and the \\ WOODS HOLE OCEANOGRAPHIC INSTITUTION
}

September 1990

(c) Wayne Richard Blanding, 1990

The author hereby grants to MIT and WHOI permission to reproduce and to distribute copies of this thesis document in whole or in part.

$1 \% \mathrm{O} \cdot \mathrm{M}$ 'SSWW ' $\exists 70 \mathrm{H}$ SOOOM

사밤 17

AYOIVAOgd7

7Ұ01507019

$\exists$ INIUVW

Signature of Author

Joint Program in Oceanographic Engineering Massachusetts Institute of Technology Woods Hole Oceanographic Institution August 10, 1990

Certified by

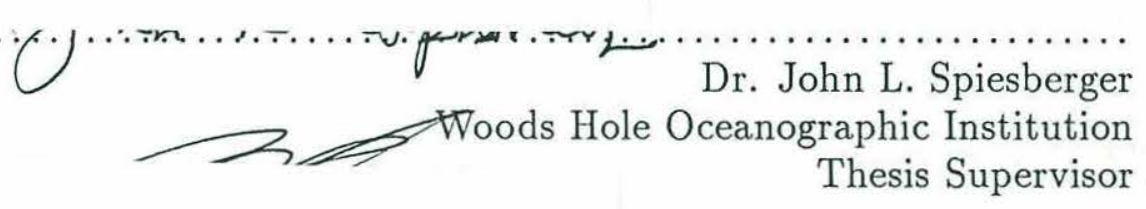

Certified by ................... re
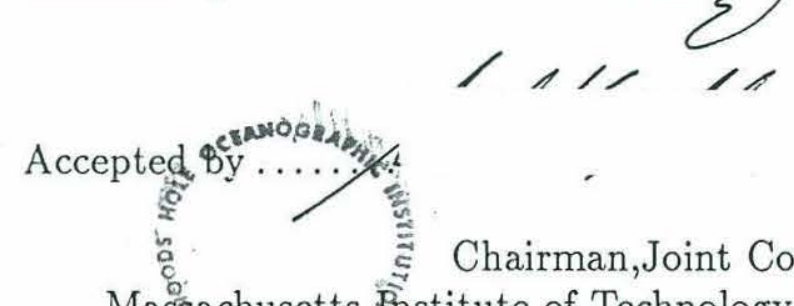

Massachusetts

Dr. Henrik Schmidt Massachusettg Institute of Technology 0 r.................... Dr. W. Kendall Melville Chairman,Joint Committee for Oceanographic Engineering 1930 


\title{
Observations of Ocean Fluctuations Between 15 and 23 Hour Periods in the Pacific \\ by
}

Wayne Richard Blanding

\author{
Submitted to the Massachusetts Institute of Technology/ \\ Woods Hole Oceanographic Institution \\ Joint Program in Oceanographic Engineering \\ on August 10, 1990, in partial fulfillment of the \\ requirements for the degree of \\ Oceanographic Engineer
}

\begin{abstract}
Pulse-like acoustic signals are transmitted from an acoustic source near Oahu to seven receivers off the west coast of the United States for a 124-day period in 1988. Acoustic travel-time oscillations are observed in the received signal at periods between 15 and 23 hours, which are caused by barotropic (or first or second mode baroclinic) fluctuations in the ocean. It is shown that these fluctuations cannot be local processes isolated to either the source or to the receivers. It is further shown that resonant barotropic gravity wave modes (Platzman et al., 1981) are not consistent with the data. The cause of these fluctuations remains unresolved, but the data and other oceanographic measurements put many constraints on the process causing these fluctuations.
\end{abstract}

Thesis Supervisor: Dr. John L. Spiesberger

Woods Hole Oceanographic Institution 


\section{Acknowledgments}

I am grateful to my advisor, Dr. John Spiesberger, who was able to make these past two years enjoyable as well as productive and without whom this thesis would not have been written.

I thank Arthur Miller, who provided me with the numerical model of the Pacific basin normal gravity modes, and Doug Luther, who provided me with sea level data.

I acknowledge the financial support of the U.S. Navy, and for their allowing me to take time from sea duty to attend MIT and spend my summers in research on Cape Cod at WHOI.

Finally, I thank Betsy Pratt for patiently drafting many of the figures used in this thesis. 


\section{Contents}

1 Introduction $\quad 8$

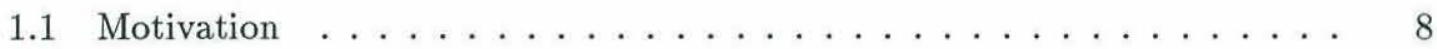

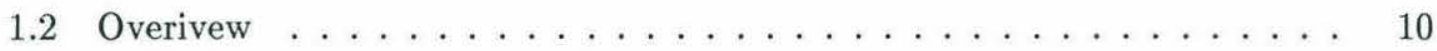

2 Acoustic Propagation $\quad 11$

3 The Experiment and Signal Processing 14

3.1 Experimental Layout . . . . . . . . . . . . . . . . . . . . . . 14

3.2 Signal Processing . . . . . . . . . . . . . . . . 16

4 Hypotheses $\quad 18$

4.1 Hypothesis 1: Ocean Fluctuations Localized to R3 . . . . . . . . . . 19

4.2 Hypothesis 2: Independent Ocean Fluctuations Near Each Receiver . . . . 19

4.3 Hypothesis 3: Oceanic Fluctuation Localized Near the Acoustic Source . . 23

4.3 .1 Island Trapped Waves . . . . . . . . . . . . . . 23

4.3.2 Other Near-Source Fluctuations . . . . . . . . . . 25

4.4 Hypothesis 4: Resonant Barotropic Gravity Wave Modes . . . . . . . . . . 25

4.4.1 Resonant Shallow Water Gravity Modes . . . . . . . . . . . . 28 
5 Discussion

5.1 Summary of Results . . . . . . . . . . . . . . . . 35

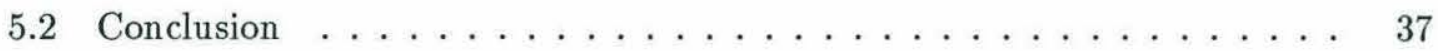

A Island Trapped Internal Waves

B Reliability of Cross-Spectral Phase

C A Two-Mode Gravity Wave Model 


\section{List of Figures}

2-1 Vertical profile of sound speed in the northeast Pacific . . . . . . . . 12

3-1 Map of acoustic tomography experiment . . . . . . . . . . . 15

4-1 Power spectral densities of the tomographic data for each receiver . . . . 20

4-2 RMS travel-times of acoustic oscillations at each receiver . . . . . . . . 21

4-3 Spectral peaks observed in 10-day tomographic records . . . . . . . . . . . 22

$4-4$ Acoustic ray path geometry near Oahu . . . . . . . . . 26

4-5 Cross-spectral phase lags for each receiver referenced to R3 . . . . . . 27

4-6 Sea level spectra at La Jolla, Calif. and Mokuoloe, Oahu . . . . . . . . . . 33

A-1 Contour integral path of scattering function . . . . . . . . . 40 


\section{List of Tables}

4.1 Modeled and observed travel-times at 15.06 hour mode . . . . . . . . 30

4.2 Modeled and observed travel-times at 18.46 hour mode . . . . . . . . . . 31

4.3 Modeled and observed travel-times at 21.66 hour mode . . . . . . . . . . 32

4.4 Measurements of sea level oscillations in the northeast Pacific Ocean . . . 34 


\section{Chapter 1}

\section{Introduction}

\subsection{Motivation}

Acoustic tomography has been successfully used to analyze mesoscale (order 100-1000km) features of the ocean (Spiesberger et al., 1983; DeFerrari and Nguyen, 1986; and others). For these experiments, other synoptic methods of extracting information about mesoscale features are available (i.e. XBT's, CTD's, and current meters). Yet when considering synoptic measurements on a basin-scale, the cost of deploying such equipment becomes prohibitive. In the past, analytic and numerical methods have been relied upon to provide dynamic models of these features. An acoustic tomography array can, at reasonable cost, be used to obtain synoptic measurements of barotropic, basin-scale oceanographic phenomena (Spiesberger et al., 1989b).

A four month basin-scale tomography experiment was performed in 1988 in the northeast Pacific where a bottom-mounted source near Oahu transmitted a continuous $(133 \mathrm{~Hz})$ acoustic signal to seven bottom-mounted receivers off the west coast of the United States. 
Signal processing converted the signal at the receiver into an equivalent signal which would be received if the source transmitted a pulse-like signal of $60 \mathrm{~ms}$ duration at $184.2 \mathrm{~s}$ intervals. Changes in acoustic travel-time are measured by estimating the phase change of the received signal of adjacent records. This experiment follows up two earlier experiments (in 1983 and 1987) in which a source near Oahu transmitted to one receiver off the coast of northern California for 5 and 21 days, respectively.

These earlier experiments yield the following results. Travel-time oscillations at tidal frequencies are observed, and are caused by barotropic tidal currents. In addition, significant oscillations are observed at many non-tidal frequencies. Specifically, at periods between 15 and 23 hours, oscillations are observed having rms variations of about $10 \mathrm{~ms}$ which vary in amplitude and frequency on about a 10-day time scale, indicating that they may be intermittently excited. Since data were only available at one receiver, it has not been possible to match these oscillations to an oceanographic process (Spiesberger et al., 1989b)

With results from the 1988 experiment, presented in this thesis, we examine four hypotheses in a search for the oceanographic source of these oscillations. The hypotheses are:

- The ocean fluctuates only near one receiver.

- The ocean fluctuates independently at each receiver.

- The ocean fluctuates only near the source.

- The ocean fluctuates at basin-scales as a result of resonant barotropic gravity wave modes. 
We show each of these hypotheses to be unlikely, and are not able to provide a theory for the cause of the observed travel-time oscillations which is consistent with both the tomographic data and other measurements of the ocean.

\subsection{Overivew}

The thesis is organized as follows. Chapter 2 contains a brief discussion of acoustic propagation in the ocean as it relates to acoustic tomography. Chapter 3 discusses the experiment and signal processing. Chapter 4 examines each of the four hypotheses proposed to explain the cause of the acoustic travel-time oscillations. Chapter 5 concludes with a discussion of the constraints placed on the problem by both the tomographic data and other measurements, and suggests other avenues of research for this problem. 


\section{Chapter 2}

\section{Acoustic Propagation}

Sound travels at about $1500 \mathrm{~m} \cdot \mathrm{s}^{-1}$ in water, which is about four and one-half times that of air. Sound speed increases with increasing temperature, increasing pressure, or, to a much smaller extent, increasing salinity. At middle and equatorial latitudes, the speed of sound initially decreases with depth because of cooling, and then increases in the deep ocean due to great pressure, resulting in a sound speed minimum at about $1 \mathrm{~km}$ depth (Figure 2-1). Application of Snell's law of refraction shows that sound is refracted towards regions of lowest sound speed (Officer, 1958). The ocean thus has a sound channel (Ewing and Worzel, 1948) which focuses acoustic energy away from the surface and bottom where significant attenuation and scattering of the signal would otherwise occur (Figure 2-1). This fact, coupled with remarkable water transparency to sound at frequencies below about $300 \mathrm{~Hz}$ (Urick, 1983), allows detection of sound propagated over thousands of kilometers.

At frequencies above about $100 \mathrm{~Hz}$, propagation of sound can be modeled with ray theory, an approximate solution of the acoustic wave equation, since the acoustic wave- 

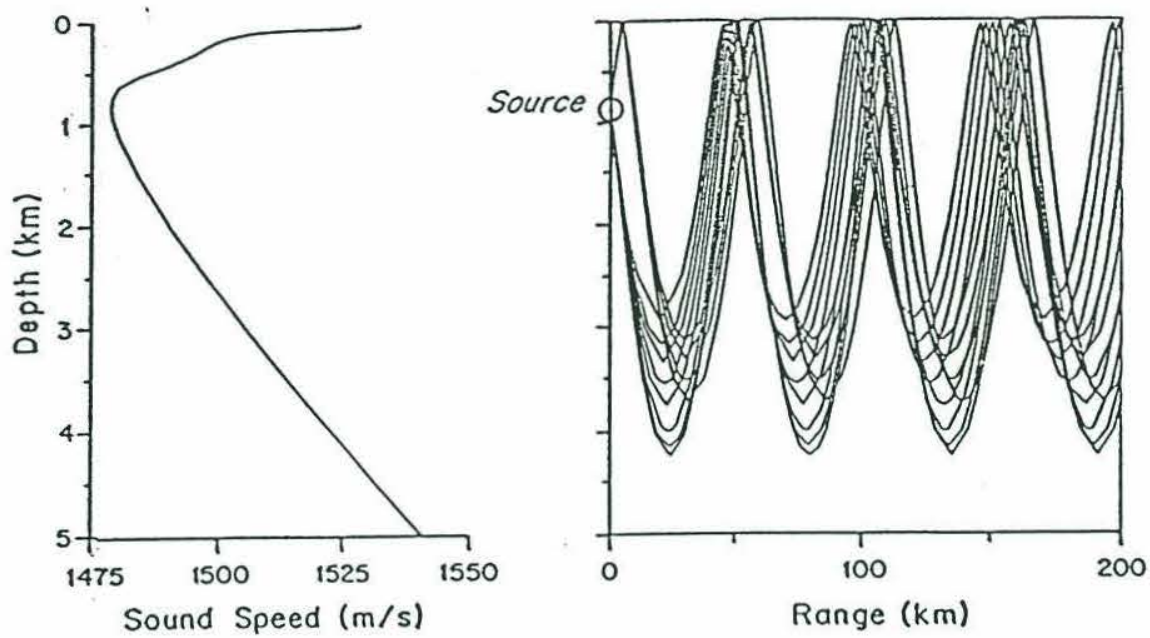

Figure 2-1: Vertical profile of sound speed in the northeast Pacific half-way between Oahu and California (left). Twelve acoustic ray paths originating from a source on the sound channel axis for a $200 \mathrm{~km}$ section of ocean (right).

length (about $15 \mathrm{~m}$ at $100 \mathrm{~Hz}$ ) is much less than the vertical scale of the channel (about 1 $\mathrm{km}$ ). Ray theory predicts that an acoustic pulse emitted from a submerged source travels to a distant receiver along ray paths (multipaths) which have different travel-times in accordance with Snell's law of refraction (Officer, 1958). Each ray path samples a different vertical slice of the ocean, based on the departure angle (from the horizontal) of the ray from the source. Thus the travel-times of different multipaths can be used in an inverse problem to solve for baroclinic fluctuations in the ocean. Alternatively, travel-times from all multipaths can be combined in an inverse problem to solve for barotropic fluctuations in the ocean.

To first order, fluctuations in acoustic travel-time can be computed by assuming that the ray path does not move in the presence of fluctuations (Munk and Wunsch, 1979). Perturbations of acoustic travel-time, $\delta \tau_{i}$, for the $i^{\text {th }}$ ray path, $\Gamma_{i}$, are primarily related 
to in-situ fluctuations of temperature, $\delta \theta$, hydrostatic pressure, $\rho g \eta$, and current, $\vec{u}$, according to,

$$
\delta \tau_{i}(t)=-\alpha \int_{\Gamma_{i}} \frac{\delta \theta(\vec{s}, t)}{c_{o}^{2}(\vec{s}, t)} d s-\beta \int_{\Gamma_{i}} \frac{\eta(\vec{s}, t)}{c_{o}^{2}(\vec{s}, t)} d s-\int_{\Gamma_{i}} \frac{\vec{u}(\vec{s}, t) \cdot \overrightarrow{d s}}{c_{o}^{2}(\vec{s}, t)}
$$

where the $i^{\text {th }}$ ray path has coordinates parameterized by $\vec{s}$ (with differential elements $d s$ and $\overrightarrow{d s}$ ), and $t$ is time. The reference sound speed is $c_{o}(\vec{s}, t)$, and that portion of the sea-surface displacement related to hydrostatic fluctuations is $\eta$. The water density is $\rho$ and the gravitational acceleration is $g$. The constants are,

$$
\begin{gathered}
\alpha \approx 4.72 \times 10^{-3} \mathrm{~km}\left({ }^{\circ} \mathrm{C} \cdot \mathrm{s}\right)^{-1} \\
\beta \approx 1.7 \times 10^{-2} \mathrm{~s}^{-1},
\end{gathered}
$$

(Spiesberger et al., 1989b).

Suppose a ray path travels a length $L(\mathrm{~km})$ in the presence of a uniform oceanic fluctuation where current (parallel to the ray path) is $u(\mathrm{~m} / \mathrm{s})$, or where the temperature fluctuation is $\delta \theta\left({ }^{\circ} \mathrm{C}\right)$, or where hydrostatic pressure changes are caused by sea-surface displacement $\eta(\mathrm{m})$. Then if currents, temperature, or sea-surface oscillations are individually responsible, the travel-time oscillation (s) is approximately,

$$
\begin{aligned}
& \delta \tau \approx-4.44 \times 10^{-4} L u \\
& \delta \tau \approx-2.1 \times 10^{-3} L \delta \theta \\
& \delta \tau \approx-7.55 \times 10^{-6} L \eta,
\end{aligned}
$$

(Spiesberger et al., 1989b). 


\section{Chapter 3}

\section{The Experiment and Signal}

\section{Processing}

\subsection{Experimental Layout}

A continuous, phase-modulated signal (center frequency $133 \mathrm{~Hz}$, bandwidth $17 \mathrm{~Hz}$ ) is transmitted between 6 January and 9 May 1988 (124 days) from a bottom-mounted source off the north coast of Oahu (183 m depth) to seven bottom-mounted receivers off the west coast of the United States (Figure 3-1). The receivers are about 3000 to $4000 \mathrm{~km}$ from the source and have acoustic travel-times of about 30 to $40 \mathrm{~min}$ respectively. The source is within $30 \mathrm{~m}$ of the sources used for the 1983 and 1987 experiments (Spiesberger et al., 1989b). 


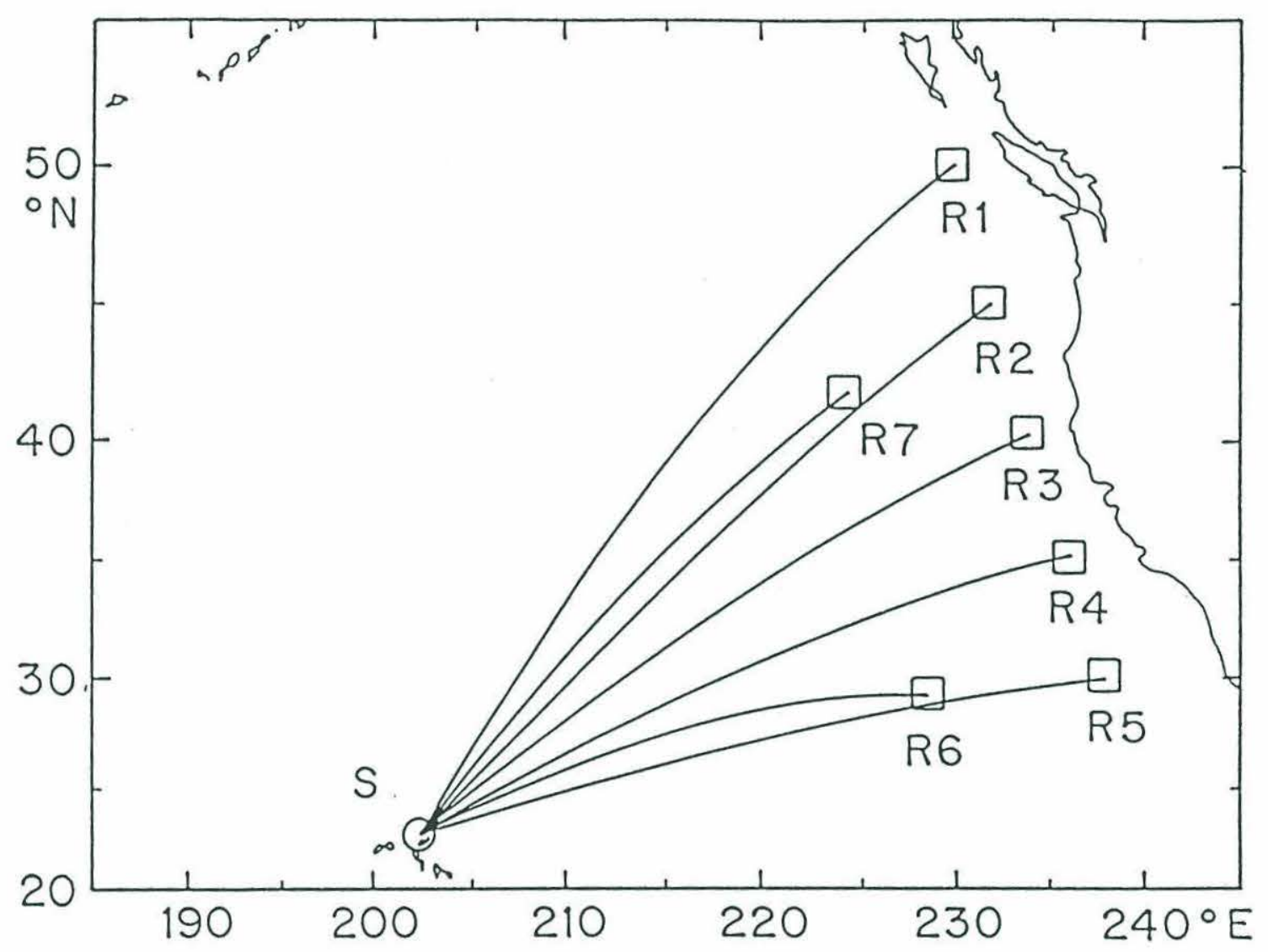

Figure 3-1: The 1988 acoustic tomography experiment consists of a bottom-mounted source (S) located near Kaneohe Bay, Oahu and seven bottom-mounted receivers (R1 to R7) whose approximate positions are shown. The distances between the source and the receivers are about 3000 to $4000 \mathrm{~km}$. 


\subsection{Signal Processing}

In order to raise signal-to-noise ratio above a detectable threshold, the transmitted signal is phase-modulated every eight cycles using a 511-digit pseudo-random code. A matched filter at each receiver compresses the energy of the $30.7 \mathrm{~s}$ cycle into that of an equivalent transmitted pulse of $60 \mathrm{~ms}$ duration. Six such cycles are averaged together, yielding traveltime records at $184.2 \mathrm{~s}$ intervals, further increasing signal-to-noise ratio. This processing is the same as that used in prior experiments (Spiesberger et al., 1989a; Metzger, 1983).

At each receiver, we measure changes in acoustic phase, $\delta \phi$, at intervals of $184.2 \mathrm{~s}$ using a maximum-likelihood estimator which computes the phase of the complex crosscorrelation of adjacent travel-time records (Spiesberger et al., 1989a). This estimator is optimally designed to detect barotropic fluctuations as well as first or second mode baroclinic fluctuations, and is designed to attenuate signals from higher vertical modes.

If the acoustic travel-time fluctuates at period $T$, then the change in travel-time is related to the change in acoustic phase by,

$$
\delta \tau=\frac{T \delta \phi}{\omega_{c} \Delta t}
$$

where the source frequency, $\omega_{c}$, equals $2 \pi \cdot 133 \mathrm{rad} \cdot \mathrm{s}^{-1}$ and the time between travel-time records, $\Delta t$, equals $184.2 \mathrm{~s}$ (Spiesberger et al., 1989a). Travel-time changes are related to oceanographic fluctuations according to Equation 2.1.

We are interested in oceanographic fluctuations other than internal waves of higher mode number $(>2)$. The acoustic signals associated with these internal waves are considered to be a portion of the "noise." The precision of the travel-time estimates $(135 \mu \mathrm{s}$ at $\Delta t=184.2 \mathrm{~s})$ is limited by the higher mode internal waves rather than by clock errors 
at the source and receiver or by acoustic noise (Spiesberger et al., 1989a). 


\section{Chapter 4}

\section{Hypotheses}

We consider four hypotheses to explain measured fluctuation in acoustic travel-time at periods between the semi-diurnal and diurnal tides. They are:

1. The ocean fluctuates only near one receiver (receiver R3 of Figure 3-1). Previous papers only analyzed data from R3 (Spiesberger et al., 1989a,b). In section 4.1, we find this hypothesis to be false.

2. The ocean undergoes independent fluctuations near each receiver. In section 4.2 , we discuss evidence which weighs against this hypothesis.

3. The ocean fluctuates only near the acoustic source. In section 4.3 , we discuss evidence which disagrees with this hypothesis.

4. The ocean fluctuates at basin-scales. In section 4.4 , we present evidence that the oscillations are not resonant barotropic gravity wave modes of the world's oceans. 


\subsection{Hypothesis 1: Ocean Fluctuations Localized to R3}

All seven receivers exhibit prominent oscillations in acoustic travel-time between the semidiurnal and diurnal tidal periods (Figures $4-1$ and $4-2$ ). Therefore this hypothesis is not true.

\subsection{Hypothesis 2: Independent Ocean Fluctuations Near} Each Receiver

We first check if acoustic travel-time oscillations of the same frequency are simultaneously observed at all receivers. The 124-day experiment is divided into twelve consecutive 10-day segments. Periodograms are computed for each receiver which collected data during the segment. We find that the oscillations between periods of 15 and 23 hours are simultaneously observed by most receivers (Figure 4-3). For example, between yeardays 70 and 79 , all seven receivers exhibit an oscillation at periods between 16.1 and 17.5 hours and exhibit an oscillation at periods between 19.0 and 20.9 hours (Figure 4-3). We conclude that most receivers simultaneously observe travel-time oscillations at the same frequency.

We believe the above data strongly weighs against hypothesis 2. Further evidence against this hypothesis is presented next.

The rms variations of acoustic travel-time are about $10 \mathrm{~ms}$ for each peak observed in the 10-day periodograms at R1 to R7 (Figure 4-3). In 1983 and 1987, acoustic os- 


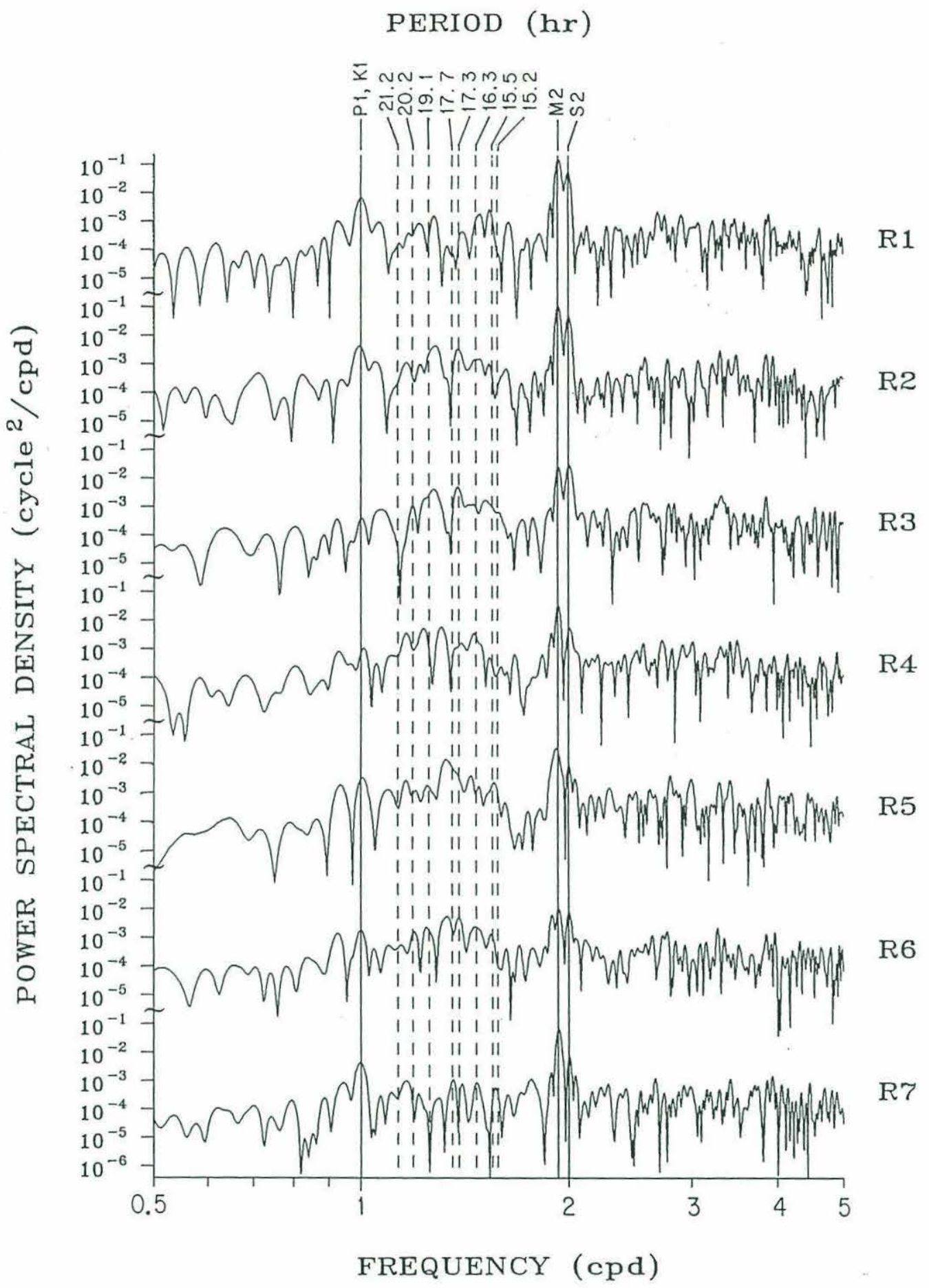

Figure 4-1: Power spectral densities of a 42-day time series (yeardays 41 to 83,1988 ) of phase differences at each receiver. A Bartlett window is applied to the data. The mean spectral level due to random walk noise (Spiesberger et al., 1989a) is at or below $1 \times 10^{-5}$ cycles $^{2} / \mathrm{cpd}$. The vertical solid lines mark prominent tidal periods. The vertical dashed lines mark periods of all resonant barotropic gravity modes with periods between 15 and 23 hours (Platzman et al., 1981). 


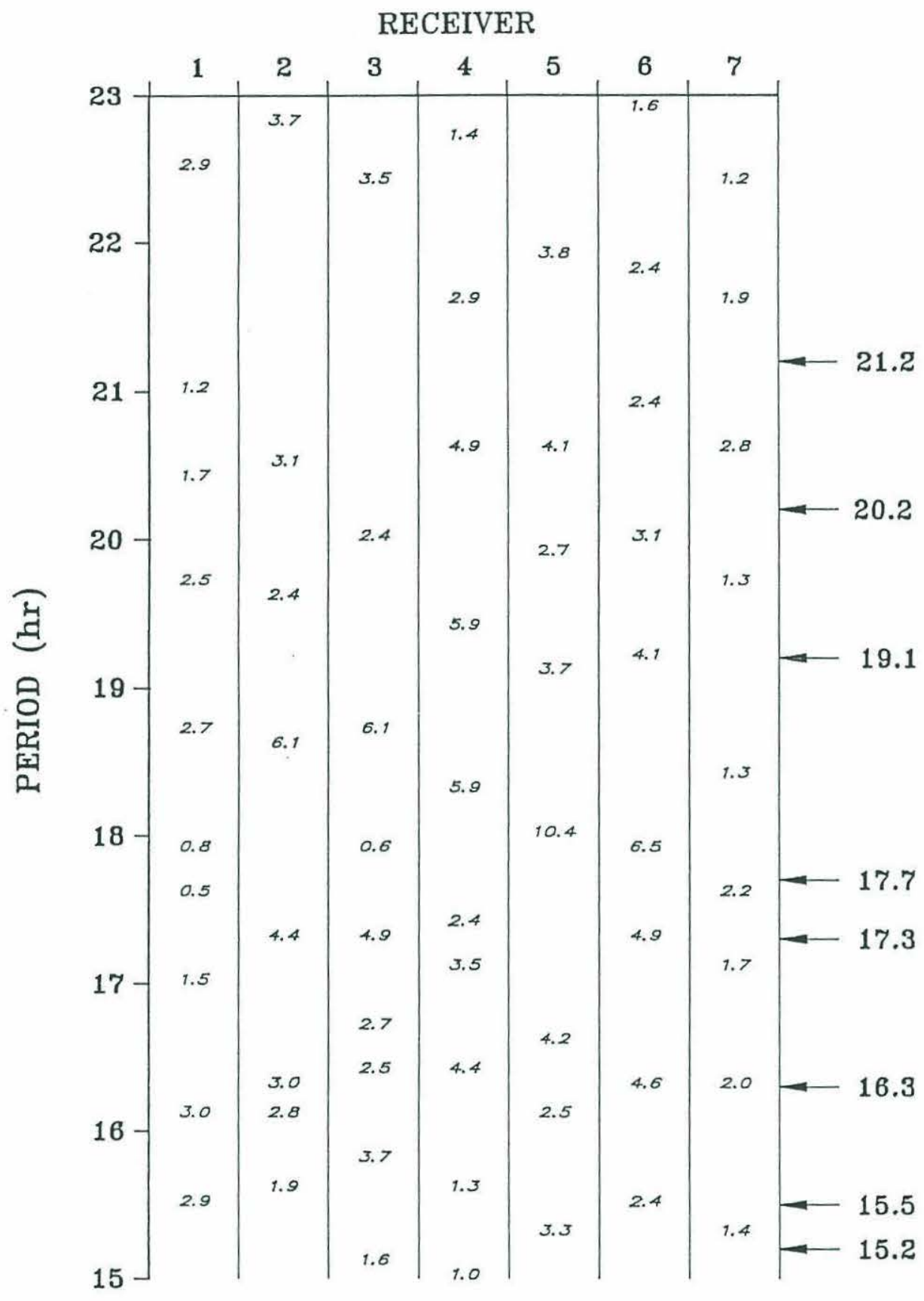

Figure 4-2: RMS travel-times (ms) are shown for the peaks in Figure 4-1 with periods between 15 and 23 hours (vertical axis). The arrows (right axis) mark the periods of all resonant barotropic gravity modes with periods between 15 and 23 hours (Platzman et al., 1981). 


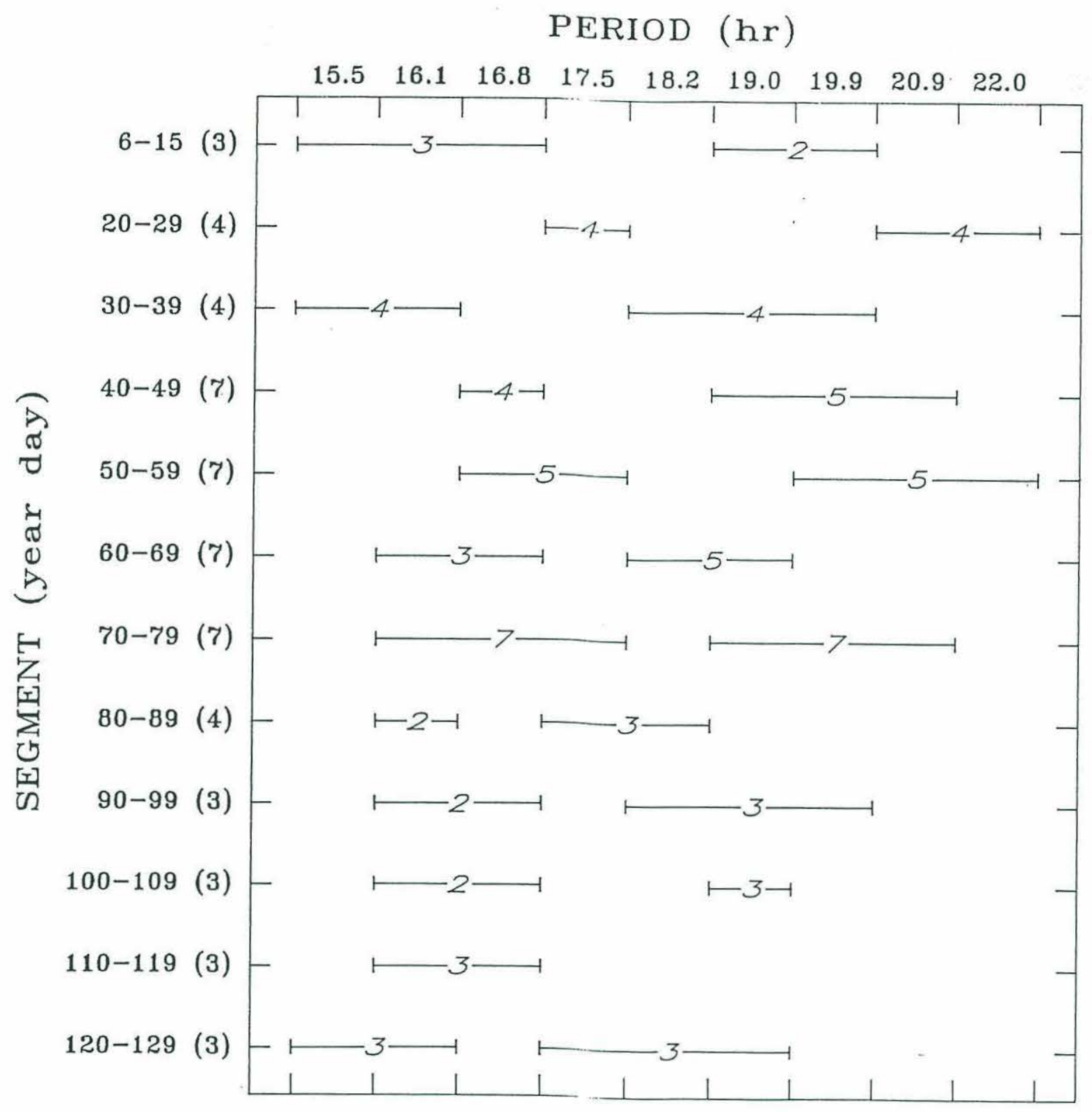

Figure 4-3: The number of receivers exhibiting spectral peaks within a given range of periods is shown for each 10-day segment of the experiment. The number of receivers for which data are available for each segment is noted in parenthesis in the left column. In each case, the spectra are computed using a Bartlett data window. The periods listed (top) are the eigenfrequencies of the periodogram. The spacing between eigenfrequencies is approximately half the resolution bandwidth of the periodogram (Harris, 1978). 
cillations near a 15 hour period had similar size (Spiesberger et al., 1989a,b). Applying Equations 2.2 and 2.3, we calculate how large a barotropic current or how large a temperature perturbation must be to yield an rms variation of $10 \mathrm{~ms}$. If the ocean fluctuations are confined within a $50 \mathrm{~km}$ region of each receiver, a uniform current of $0.6 \mathrm{~m} \cdot \mathrm{s}^{-1}$ or a uniform temperature perturbation of $0.132^{\circ} \mathrm{C}$ is required. Magnitudes of the perturbation in current and temperature are inversely proportional to the horizontal scale of the fluctuation. If the fluctuation is confined to $25 \mathrm{~km}$, then the associated current or temperature perturbation would be twice the above values. The magnitudes of these perturbations are implausible.

We therefore find it unlikely that independent ocean fluctuations near each receiver give rise to the observed oscillations in acoustic travel-time at periods between the semidiurnal and diurnal tides.

\subsection{Hypothesis 3: Oceanic Fluctuation Localized Near the}

\section{Acoustic Source}

We present two results which indicate that the acoustic oscillations with periods between 15 and 23 hours are not caused by ocean fluctuations confined to the region of the acoustic source. These results are presented separately in subsections 4.3 .1 and 4.3 .2 below.

\subsubsection{Island Trapped Waves}

A model for the trapping of super-inertial internal waves around islands was proposed by Wunsch (1972) to explain thermal fluctuations near Bermuda. In this model the 
ocean (constant depth, rigid lid), containing a cylindrical island, is realistically stratified allowing for free internal waves. Internal wave scattering exhibits pseudo-resonances if the period of the incident wave matches the free wave travel-time around the cylinder (island). When this model is applied to Oahu (Appendix A), no significant pseudo-resonances are observed for wave periods between 10 and 25 hours.

A second mechanism for the generation of island trapped waves, refraction (resulting in "trapped-leaky" waves), is not likely to occur around Oahu due to the narrow shelf width and complicated geometry of the island (Luther, 1985; Longuet-Higgins, 1967; Summerfield, 1972). Thus, available theoretical models fail to provide a mechanism for the generation of island trapped waves around Oahu.

Even though we find no theoretical justification for island trapped waves, these theories are only approximations which contain some questionable assumptions, and such waves may exist. For example, Luther (1985) has analyzed 10- and 14-year records of sea-level at two stations on opposite sides of Oahu (Mokuoloe and Honolulu). His analysis reveals peaks in the sea level spectra from each station at periods of 17.0 and 20.0 hours with amplitudes of about $0.5 \mathrm{~cm}$. Cross-spectral phase relationships suggest that these spectral peaks could result from island trapped waves with horizontal wavelengths equal to 1 or $\frac{1}{2}$ the circumference of Oahu. Patzert and Wyrtki (1974) have observed clockwise currents of about $5 \mathrm{~cm} / \mathrm{s}$ around Oahu, providing further support for the existence of island trapped waves.

If there is an island trapped wave of the type hypothesized by Luther, this is not the wave observed in the tomographic measurements. Consider the transmission paths to R3 and R4 which leave the source at bearing angles differing by about $10^{\circ}$ (Figure 
4-4). If a 15.5 hour period island trapped wave has a horizontal wavelength of $\frac{1}{2}$ the island circumference, then the predicted phase lag between acoustic signals at R3 and R4 is $5^{\circ}$ (Figure 4-4). Cross-spectral phases at 15.5 hour period (at R3 and R4) have values of $157^{\circ} \pm 15^{\circ}$ (Figure 4-5 and Appendix B). Calculations of cross-spectral phases at other receiver combinations and periods yield results which are also inconsistent with the island trapped wave hypothesis of low horizontal mode number (Figure 4-5). If the horizontal mode number of the island trapped wave is 64 , then the predicted phase lag at R3 and R4 would match the observed phase lag. We do not believe that realistic coastlines and bathymetric features could support such an island trapped wave. The tomographic measurements are therefore inconsistent with the island trapped wave hypothesis.

\subsubsection{Other Near-Source Fluctuations}

Unrealistically large fluctuations of current and temperature are required to yield acoustic travel-time oscillations of $10 \mathrm{~ms} \mathrm{rms}$ (Section 4.2). We conclude that the tomographic measurements are inconsistent with hypothesis 3 .

\subsection{Hypothesis 4: Resonant Barotropic Gravity Wave Modes}

We investigate the hypothesis that the acoustic oscillations are caused by resonant barotropic gravity wave modes. 


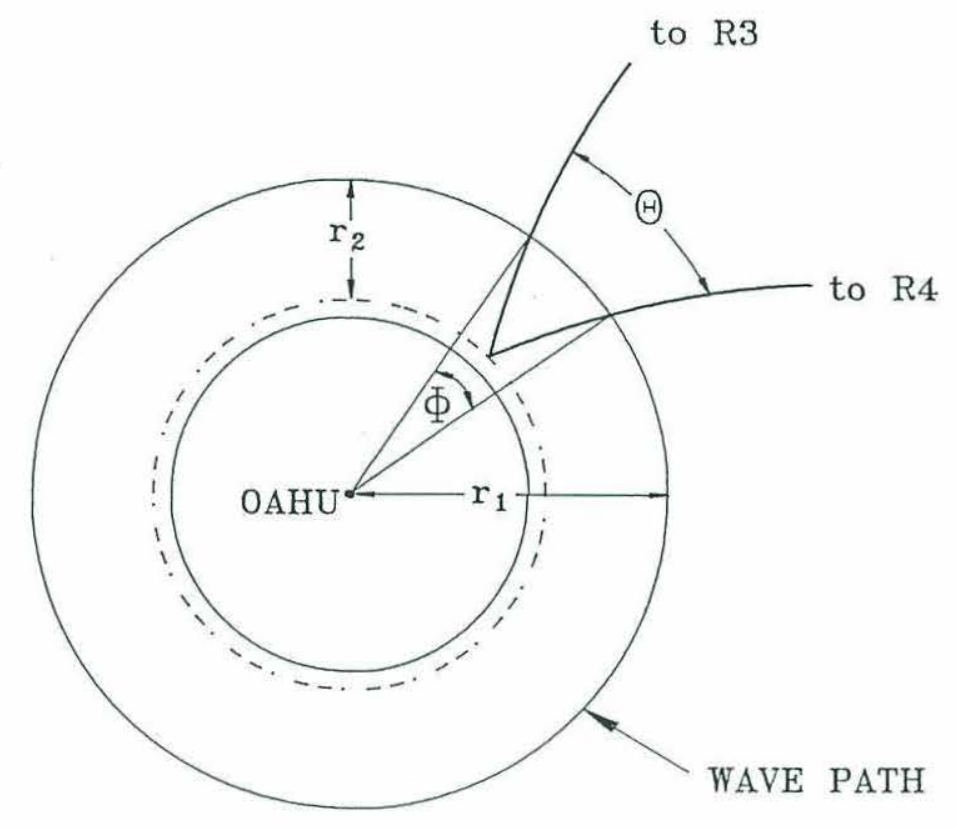

Figure 4-4: The relationship is shown between the phase lag, $n \Phi$, of an island trapped wave (horizontal mode $n$ ) at the wave's intersection with two ray paths and the difference in bearing angle of the ray paths, $\Theta$. The tomographic signal generated by an island trapped wave will have a phase lag between the two receivers equal to about $n \Phi$, where $n \Phi=n \frac{r_{2}}{r_{1}} \Theta$. The distances $r_{1}$ and $r_{2}$ are about 40 and $10 \mathrm{~km}$ respectively, and $\Theta$ is about $10^{\circ}$. The angles in this figure are enlarged for clarity. 


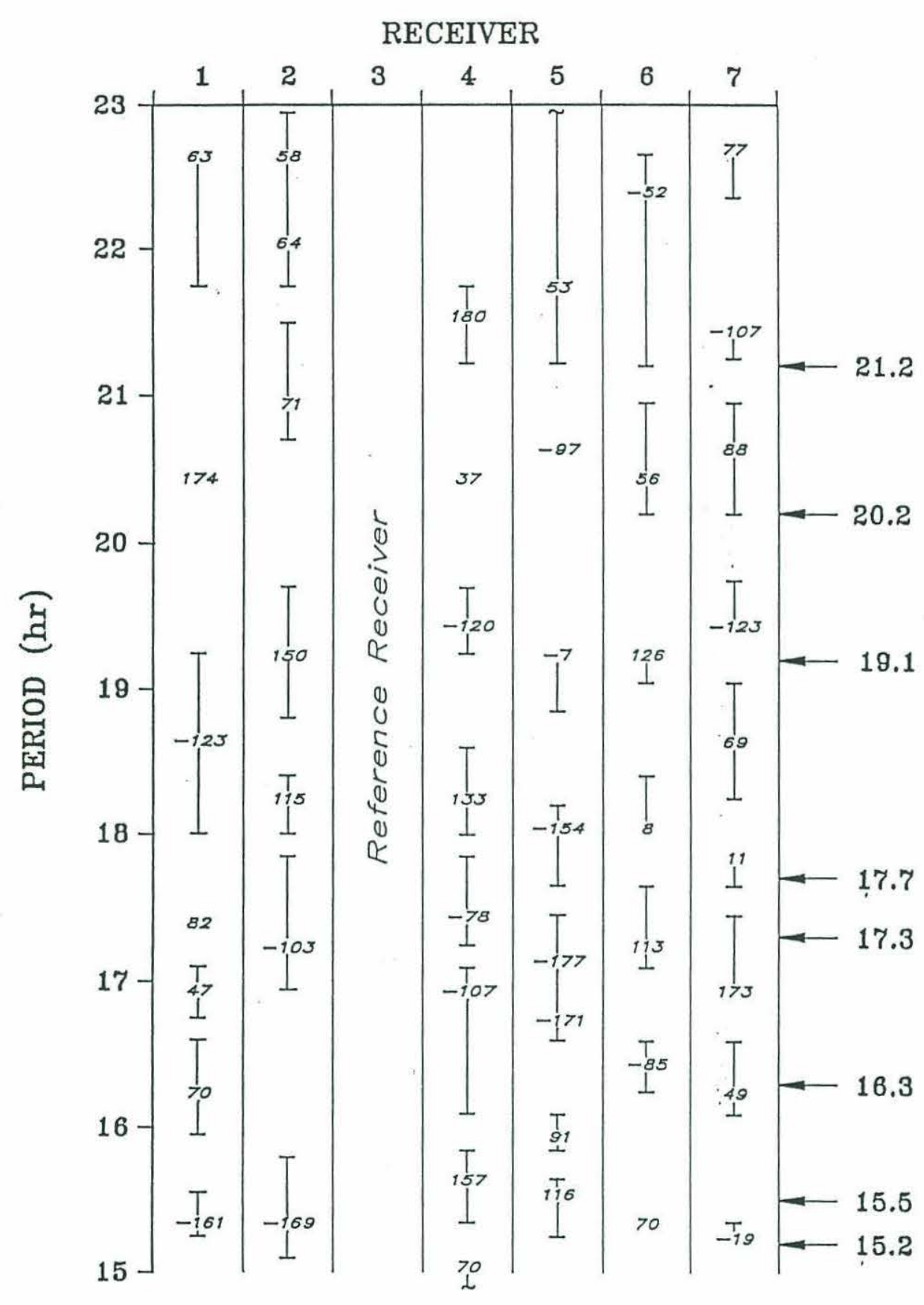

Figure 4-5: Phase lags (degrees) are shown for periods at which coherency peaks occur in the cross-spectral estimates of 42-day time series of phase differences at each receiver referenced to R3. Each time series has the same reference time and runs from yearday 41 to 83,1988 . The error bars show the range of periods over which the phase shift remains within $\pm 15^{\circ}$ of its value at the coherency peak (Appendix B). The arrows (right axis) mark the periods of all resonant barotropic gravity modes with periods between 15 and 23 hours (Platzman et al., 1981). 


\subsubsection{Resonant Shallow Water Gravity Modes}

Platzman (1978) and Platzman et al. (1981) developed a model identifying normal modes of the world ocean. In this model, the primitive equations are applied to a finite element discretization of the world ocean (about $900 \mathrm{~km}$ between nodes) yielding eigenfrequencies of resonant barotropic vorticity and gravity modes. Corresponding to each eigenfrequency is a set of eigenvalues describing the sea level and velocity structure of each mode. Platzman et al. (1981) find eight gravity modes at periods between 15 and 23 hours (Figures 4-1, 4-2, and 4-5), two of which (15.5 and 21.2 hours) are dominant in the Pacific Ocean.

In our study, we use the same programs developed by Platzman to identify resonant, barotropic gravity modes for the Pacific Ocean only (Miller et al., 1988). Using the same grid as Platzman (with minor variations at boundary nodes), we find three resonant modes at periods between 15 and 23 hours (15.06, 18.46, and 21.66 hours). Considering the altered boundary nodes, this is consistent with the Platzman's results.

For each mode, we compute the predicted acoustic travel-time oscillation, at receiver $i$, using Equation 2.1 and obtain results of the form,

$$
\delta \tau_{i}(t)=\alpha a_{i} \sin \left( \pm \sigma t+\phi_{i}+\zeta\right)
$$

where $a_{i}$ and $\phi_{i}$ are the amplitude and phase of the acoustic travel-time computed from the model, $\sigma$ is frequency and $t$ is time. Since the model magnitude and phase of the seasurface and velocity are based on arbitrary references, scaling factors $\alpha$ and $\zeta$ are required when comparing the predicted and observed acoustic travel-time oscillations. For each mode, $\zeta$ is chosen such that the phase of the predicted acoustic travel-time at R3 (the reference receiver) is $0^{\circ}$, and $\alpha$ is chosen such that for the amplitude of the predicted 
acoustic travel-time matches that of R3. We estimate the accuracy of our model travel times to be about $20 \%$ in magnitude and $\pm 15^{\circ}$ in phase.

A comparison of the observed and predicted acoustic travel-times at each receiver are shown in Tables $4.1,4.2$, and 4.3 for each of the three modes. The model phase at each mode deviates significantly from the observed phase. For example, at the 15.06 hour

mode there is a consistent $-160^{\circ}$ discrepancy between the model and the observations. We conclude that the observed travel-time oscillations are not caused by a single mode.

We investigate the case where two modes occur simultaneously at each modal frequency (clockwise and counterclockwise). We find no combination of these modes at any of the three modal frequencies yield the observed phase and magnitude of acoustic traveltime oscillations at each receiver (Appendix C). We therefore conclude that the two-mode hypothesis is not consistent with the observations.

\subsubsection{Comparison With Other Measurements}

For each resonant barotropic shallow water gravity mode modeled in section 4.4 .1 , we observe that in order to yield an averaged (42-day) rms acoustic travel-time oscillation of about $3 \mathrm{~ms}$, sea surface oscillations of about $5 \mathrm{~cm}$ are required. However, other observations of the sea-surface at these periods and record lengths exhibit magnitudes which are about ten times less (Table 4.4). Errors from boundary condition approximation and discretization cannot account for this difference. Based on this and on the failure of the model to fit the data, we conclude that the tomographic oscillations observed are not the result of resonant barotropic gravity wave modes of the Pacific Ocean. 


\begin{tabular}{|c|c|c|}
\hline \multicolumn{3}{|c|}{ 15.06 Hour Mode } \\
\hline Receiver & $\begin{array}{c}\text { Observed } \\
\text { Amplitude (ms) }\end{array}$ & $\begin{array}{c}\text { Model } \\
\text { Amplitude (ms) }\end{array}$ \\
\hline 1 & 2.9 & 2.0 \\
2 & 1.9 & 2.2 \\
3 & $3.7 / 1.6$ & 1.6 \\
4 & $1.3 / 1.0$ & 1.3 \\
5 & 3.3 & 2.0 \\
6 & 2.4 & 1.4 \\
7 & 1.4 & 1.7 \\
\hline
\end{tabular}

\begin{tabular}{|c|c|c|c|c|c|}
\hline \multicolumn{6}{|c|}{ 15.06 Hour Mode } \\
\hline \multirow[b]{2}{*}{ Receiver } & \multirow[b]{2}{*}{$\begin{array}{c}\text { Observed Phase } \\
\text { Lag (deg.) }\end{array}$} & \multicolumn{2}{|c|}{ Positive Frequency } & \multicolumn{2}{|c|}{ Negative Frequency } \\
\hline & & $\begin{array}{l}\text { Model Phase } \\
\text { Lag (deg.) }\end{array}$ & $\begin{array}{l}\text { Deviation } \\
\text { (deg.) }\end{array}$ & $\begin{array}{l}\text { Model Phase } \\
\text { Lag (deg.) }\end{array}$ & $\begin{array}{l}\text { Deviation } \\
\text { (deg.) }\end{array}$ \\
\hline 1 & -161 & 42 & -157 & -42 & 119 \\
\hline 2 & -169 & 29 & -162 & -29 & 140 \\
\hline 3 & 0 & 0 & 0 & 0 & 0 \\
\hline 4 & 70 & -70 & -140 & 70 & 0 \\
\hline 5 & $91 / 116$ & -92 & $177 / 152$ & 92 & $1 /-24$ \\
\hline 6 & $70 /-86$ & -97 & $-167 /-11$ & 97 & $27 /-177$ \\
\hline 7 & $-19 /-167$ & 49 & $68 /-144$ & -49 & $-30 / 144$ \\
\hline
\end{tabular}

Table 4.1: Comparison of acoustic travel-time amplitudes (top) and phases (referenced to R3) (bottom) predicted by the resonant barotropic gravity wave model and those observed in the tomographic data at the 15.06 hour mode (Miller et al., 1988). Phase deviations are determined by subtracting the observed phase lag from the model phase lag. Observed phases are modulated by $\pm 360^{\circ}$ if necessary to obtain deviations between $\pm 180^{\circ}$ ( $\mathrm{a}-175^{\circ}$ deviation is only $10^{\circ}$ different from a $+175^{\circ}$ deviation). Positive and negative frequency indicates clockwise and counterclockwise wave propagation. Phase lags are accurate to $\pm 15^{\circ}$ (Appendix B). When two auto- or cross-spectral peaks occur near the predicted mode period, both are tabulated. 


\begin{tabular}{|c|c|c|}
\hline \multicolumn{3}{|c|}{ 18.46 Hour Mode } \\
\hline Receiver & $\begin{array}{c}\text { Observed } \\
\text { Amplitude (ms) }\end{array}$ & $\begin{array}{c}\text { Model } \\
\text { Amplitude (ms) }\end{array}$ \\
\hline 1 & 2.7 & 5.4 \\
2 & 6.1 & 5.4 \\
3 & 6.1 & 6.1 \\
4 & 5.9 & 7.2 \\
5 & 10.4 & 8.7 \\
6 & 6.5 & 5.2 \\
7 & 1.3 & 4.4 \\
\hline
\end{tabular}

\begin{tabular}{|c|c|c|c|c|c|}
\hline \multicolumn{6}{|c|}{ 18.46 Hour Mode } \\
\hline \multirow[b]{2}{*}{ Receiver } & \multirow[b]{2}{*}{$\begin{array}{l}\text { Observed Phase } \\
\text { Lag (deg.) }\end{array}$} & \multicolumn{2}{|c|}{ Positive Frequency } & \multicolumn{2}{|c|}{ Negative Frequency } \\
\hline & & $\begin{array}{l}\text { Model Phase } \\
\text { Lag (deg.) }\end{array}$ & $\begin{array}{l}\text { Deviation } \\
\text { (deg.) }\end{array}$ & $\begin{array}{l}\text { Model Phase } \\
\text { Lag (deg.) }\end{array}$ & $\begin{array}{l}\text { Deviation } \\
\text { (deg.) }\end{array}$ \\
\hline 1 & -123 & 8 & 131 & -8 & 115 \\
\hline 2 & $150 / 115$ & 9 & $-141 /-106$ & -9 & $-159 /-124$ \\
\hline 3 & 0 & 0 & 0 & 0 & 0 \\
\hline 4 & $-120 / 132$ & -9 & $111 /-141$ & 9 & $129 /-123$ \\
\hline 5 & $-6 /-154$ & -15 & $-9 / 139$ & 15 & $21 / 169$ \\
\hline 6 & $126 / 8$ & -18 & $-144 /-26$ & 18 & $-108 / 10$ \\
\hline 7 & 69 & 4 & -65 & -4 & -73 \\
\hline
\end{tabular}

Table 4.2: Same as Table 4.1, except comparisons are made at the predicted 18.46 hour mode. 


\begin{tabular}{|c|c|c|}
\hline \multicolumn{3}{|c|}{ 21.66 Hour Mode } \\
\hline Receiver & $\begin{array}{c}\text { Observed } \\
\text { Amplitude (ms) }\end{array}$ & $\begin{array}{c}\text { Model } \\
\text { Amplitude (ms) }\end{array}$ \\
\hline 1 & - & 3.8 \\
2 & - & 3.9 \\
3 & - & 3.5 \\
4 & 2.9 & 2.9 \\
5 & 3.8 & 2.4 \\
6 & 2.4 & 2.2 \\
7 & 1.9 & 3.3 \\
\hline
\end{tabular}

\begin{tabular}{|c|c|cc|cc|}
\hline \multicolumn{5}{|c|}{ 21.66 Hour Mode } \\
\hline \multirow{2}{*}{ Receiver } & $\begin{array}{c}\text { Observed Phase } \\
\text { Lag (deg.) }\end{array}$ & $\begin{array}{c}\text { Positive Frequency } \\
\text { Model Phase } \\
\text { Lag (deg.) }\end{array}$ & $\begin{array}{c}\text { Deviation } \\
\text { (deg.) }\end{array}$ & $\begin{array}{c}\text { Model Phative Frequency } \\
\text { Lag (deg.) }\end{array}$ & $\begin{array}{c}\text { Deviation } \\
\text { (deg.) }\end{array}$ \\
\hline & & & & & $-59 /-170$ \\
1 & $63 / 174$ & -4 & $67 / 178$ & 4 & $-56 /-69$ \\
2 & $58 / 71$ & -2 & $-60 /-73$ & 2 & 0 \\
3 & 0 & 0 & 0 & 0 & $-40 / 175$ \\
4 & $35 / 180$ & 5 & $-30 /-175$ & -5 & -67 \\
5 & 53 & 14 & -39 & -14 & 43 \\
6 & -53 & 10 & 63 & -10 & $-69 / 115$ \\
7 & $77 /-107$ & -8 & $-85 / 99$ & 8 & \\
\hline
\end{tabular}

Table 4.3: Same as Table 4.1, except comparisons are made at the predicted 21.66 hour mode. 


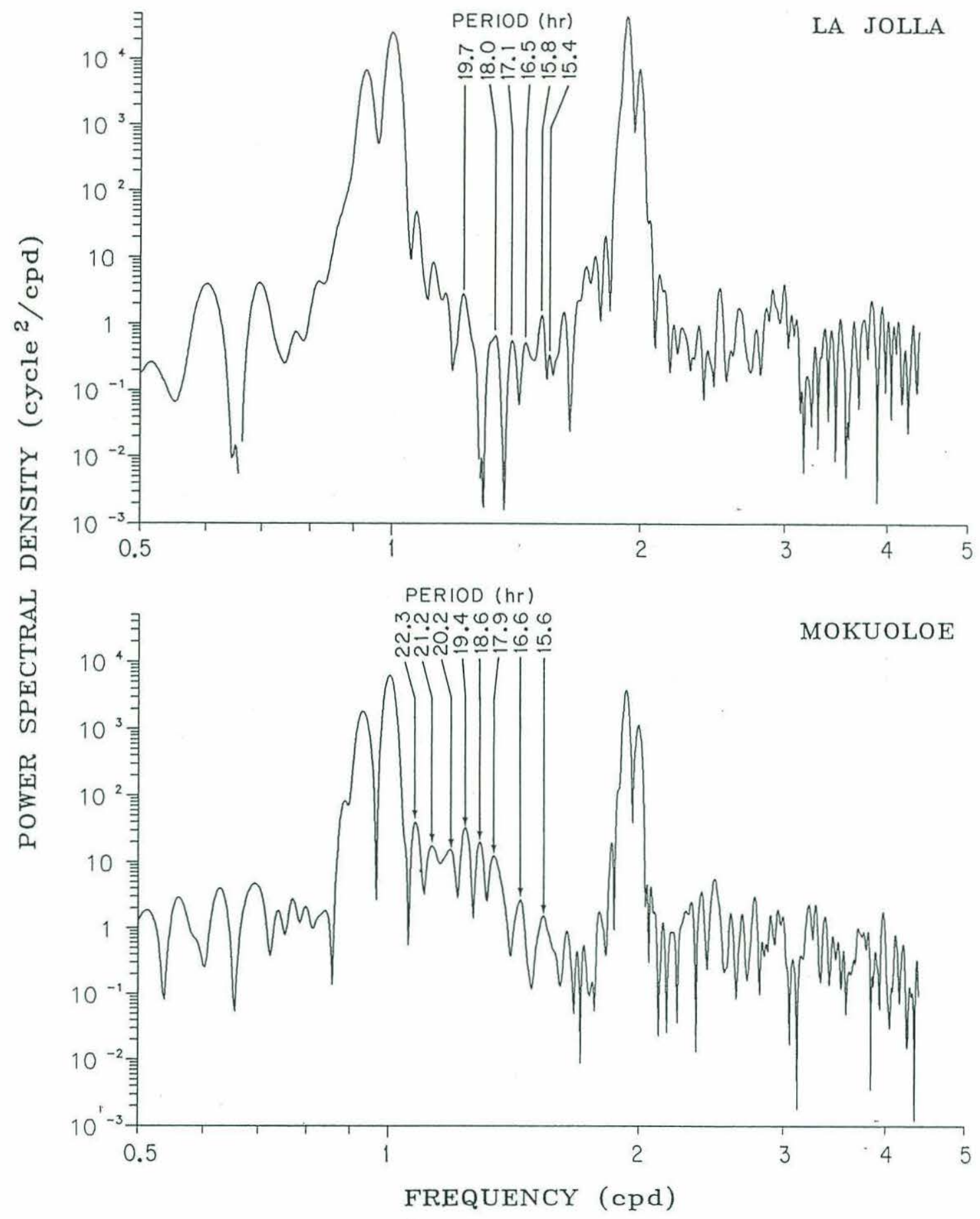

Figure 4-6: Power spectral densities of 42-day time series of sea level at La Jolla, California (top) and Mokuoloe, Oahu (bottom). The La Jolla sea level data is from yeardays 41 to 83, 1981 and the Mokuoloe sea level data is from yeardays 19 to 61,1988 . A Bartlett window is applied to the data. The labeled peaks at periods between 15 and 23 hours are not sidelobes of the tides. 


\begin{tabular}{|c|c|c|}
\hline Source (Reference) & $\begin{array}{c}\text { Inter-tidal } \\
\text { Peaks Observed? }\end{array}$ & $\begin{array}{l}\text { RMS Amplitude } \\
\text { Oscillation }\end{array}$ \\
\hline Oahu sea-level 10-year record (Luther, 1985) & Yes & $0.5 \mathrm{~cm}$ \\
\hline Oahu sea-level 42-day record (Figure 4-6) & Yes & $0.5 \mathrm{~cm}$ \\
\hline $\begin{array}{l}\text { La Jolla, Calif. sea-level 42-day record } \\
\text { (Figure 4-6) }\end{array}$ & Yes & $0.2 \mathrm{~cm}$ \\
\hline $\begin{array}{l}\text { Bottom pressure gage }(300 \mathrm{~km} \text { east of Oahu) } \\
60 \text {-day record (Filloux, 1981) }\end{array}$ & Yes & $0.1 \mathrm{~cm}$ \\
\hline $\begin{array}{l}\text { Bottom pressure gage }(1150 \mathrm{~km} \text { west of Calif.) } \\
\text { 7-day record (Filloux, 1971) }\end{array}$ & No & - \\
\hline
\end{tabular}

Table 4.4: A summary showing amplitudes of sea level oscillations above background spectral levels at periods between 15 and 23 hours. 


\section{Chapter 5}

\section{Discussion}

\subsection{Summary of Results}

We have shown in the previous chapter how the tomographic data provides several constraints on the problem of identifying a cause for the observed travel-time oscillations. Other experiments in the Pacific Ocean add further constraints. At this point, having eliminated the more obvious processes which could cause these oscillations, it is useful to summarize these constraints and perhaps obtain a new perspective on the nature of an oceanographic process required to generate the observed results. Such a process must:

- have about $0.5 \mathrm{~cm}$ oscillations in sea level.

- generate, through current or temperature perturbations, acoustic travel-times of about $10 \mathrm{~ms}$ rms over 10 -day time periods and about $3 \mathrm{~ms}$ rms over 42 -day time periods. 
- result in large phase lags in acoustic travel-time between different receivers consistent with the data.

- be intermittent (averaging over time reduces the magnitude of the oscillations).

- occur simultaneously at periods between 15 and 23 hours at similar magnitudes.

The requirement for small sea level variations brings us back to the concept of internal waves. Because of the signal processing, our data is insensitive to higher order internal wave modes, and we have previously ignored such processes. There is a possibility that internal waves at the turning latitude (where wave frequency equals the inertial frequency) may intensify and give rise to temperature perturbations sufficient to yield the observed travel-time magnitudes (M. Briscoe, personal communication).

The inertial period in the region of the experiment ranges between 32.7 hours (at Oahu) and about 15.7 hours (at R1). In order for travel-time fluctuations to occur at a receiver due to this process, the ray path must cross the turning latitude for a particular period. For example, at R5 and R6 (inertial period about 24 hours), we expect to observe fluctuations due to this process only between 24 and 32.7 hour periods. Yet we observe prominent oscillations at these receivers at 15 to 23 hour periods. Any test of this theory must account for these oscillations.

For completeness, we note in the literature that several other theories explaining the observed travel-time oscillations have been examined and rejected (Spiesberger et al., 1989b). 


\subsection{Conclusion}

We must confess that, while the results presented here are significant, they are unsatisfying in that we are unable to find an oceanographic process which explains the acoustic traveltime oscillations. Had it not been for other work demonstrating agreement between the tomographic data and models of barotropic tides (Headrick, 1990), we may have even questioned the validity of the data itself. In one sense, this study highlights the concept that while it is easy to design an experiment to prove or disprove a specific theory, it is much more difficult to find a theory to fit observed phenomena.

To return to the initial motivation for basin-scale acoustic tomography, that of obtaining synoptic measurements of basin-scale phenomena, we find ourselves both covering new ground and old ideas. Tomographic measurements provide a unique window into the understanding of basin-scale processes. Many such processes have been theorized, but have no direct observational support. Yet while trying to understand these previously unobserved phenomena, we can also obtain a fuller understanding of "well known" phenomena (such as tides).

In this study, we have limited ourselves to a class of interesting observations in a very narrow frequency range. The data set however, allows for observations of processes having periods from about 1000 hours to 6 minutes. Use of this data in conjunction with point measurements in the Pacific is a worthwhile and rich topic of research. 


\section{Appendix A}

\section{Island Trapped Internal Waves}

The solution for the perturbed pressure field of internal waves scattering from a cylinder is,

$$
P(r, \theta)=A \sum_{m=-\infty}^{\infty} \imath^{m}\left[J_{m}(k r)-\left(\frac{k a J_{m}^{\prime}(k a)-\frac{f m}{\sigma} J_{m}(k a)}{k a H_{m}^{\prime}(k a)-\frac{f m}{\sigma} H_{m}(k a)}\right) H_{m}(k r)\right] e^{\imath m \theta}
$$

where the primed variables denote derivatives, $J_{m}$ and $H_{m}$ are Bessel and Hankel functions of the first kind of order $m$ respectively, $A$ is the amplitude of the incident plane internal wave with frequency $\sigma$ and horizontal wavenumber $k, a$ is the cylinder radius, and $f$ is the Coriolis frequency (Proudman, 1914; Wunsch, 1972). The origin of the cylindrical coordinate system, with radius $r$ and polar angle $\theta$, is the center of the cylinder. The horizontal wavenumber is related to the frequency through the dispersion relationship for internal waves by

$$
k^{2}=\frac{\sigma^{2}-f^{2}}{g h_{n}}
$$

where $h_{n}$ is the equivalent depth of the $n^{\text {th }}$ internal wave mode, and $g$ is gravitational acceleration. Pseudo-resonances occur if proper choices of $k$ and $\sigma$ result in $|P(r, \theta)| / A$ 
greater than about 5 for a given cylinder radius.

A logical choice for selecting the cylinder radius is made by matching the circumference of Oahu to that of a cylinder $(29.4 \mathrm{~km})$ (Luther, 1985). Geographical considerations limit the maximum island radius to $40 \mathrm{~km}$, at which point the cylinder would touch the neighboring island of Molokai. Although not likely, bathymetry suggests the possibility of the Oahu/Molokai/Maui group of islands being considered as a single model island, resulting in a cylinder radius of about $110 \mathrm{~km}$.

Historical temperature and salinity profiles near Oahu are used to determine the equivalent depths of the first three internal wave modes $(.88 \mathrm{~m}, .25 \mathrm{~m}$, and $.10 \mathrm{~m}$ respectively). We evaluate $|P(r, \theta)|$ for equivalent depths of $.01-1.0 \mathrm{~m}$ in $.01 \mathrm{~m}$ increments and island radii of $15-50 \mathrm{~km}$ in $5 \mathrm{~km}$ increments as well as $90-120 \mathrm{~km}$ in $10 \mathrm{~km}$ increments. For each possible pairing of these equivalent depths and island radii, we evaluate the magnitude of the scattering function between 10 and 25 hours at $.28 \mathrm{hr}$ increments. The value of $|P(r, \theta)| / A$ does not exceed 3.0 and thus, no significant pseudo-resonances occur.

We also search for poles of $P(r, \theta)$ in the complex frequency plane near the real frequency axis by evaluating a contour integral (Figure A-1) for the same island radii and equivalent depth combinations used in the previous calculation. These contour integrals are all zero, indicating $P(r, \theta)$ contains no poles which cause pseudo-resonances at periods between 10 and 25 hours. 


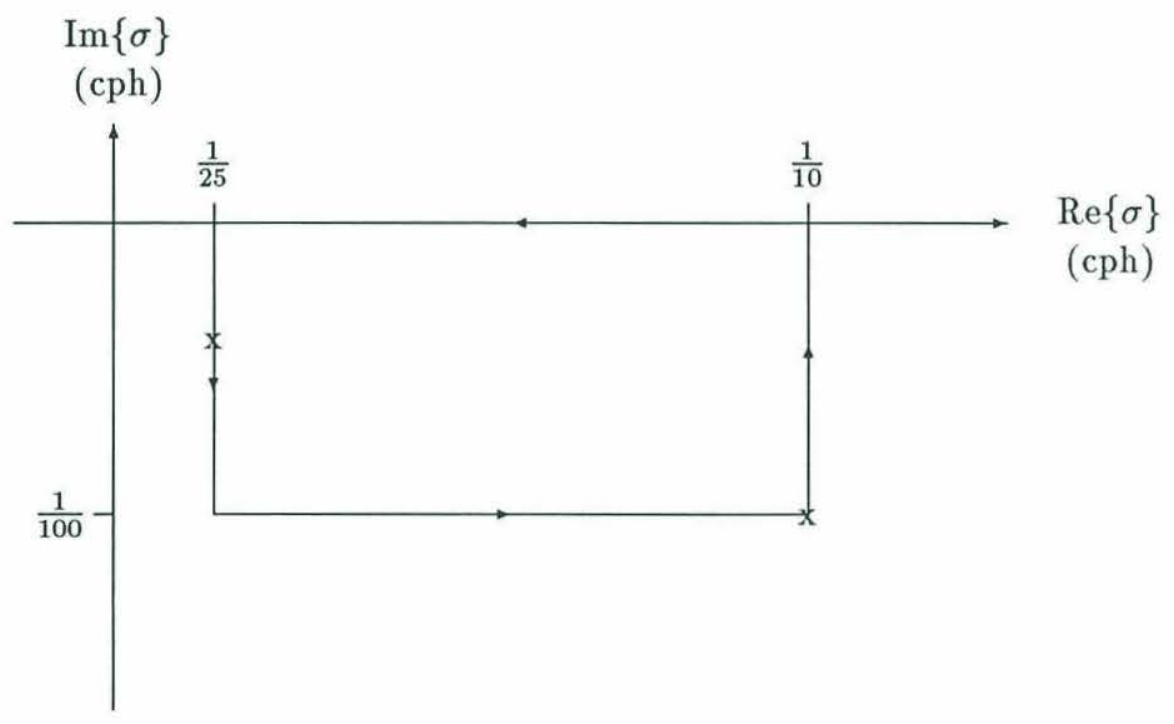

Figure A-1: The contour integral path of $P(r, \theta)$ encloses a rectangular area in the complex frequency domain, where the real axis represents the domain of pure oscillatory waves. In order for a pole of $P(r, \theta)$ to cause a pseudo-resonance at periods between 10 and 25 hours, it must lie within the region bounded by $Q>5$, where $Q$ is the resonance factor for a lightly damped oscillator $\left(Q=\frac{1}{2} \operatorname{Re}\{\sigma\} / \operatorname{Im}\{\sigma\}\right)$. Points on the contour where $Q=5$ are marked with an $\mathrm{x}$. 


\section{Appendix B}

\section{Reliability of Cross-Spectral}

\section{Phase}

We treat auto- and cross-spectral peaks as isolated events which are too large to be caused by measurement error (Spiesberger et al, 1989b). In other words, we are not interested in standard calculations of $95 \%$ confidence intervals because we do not care if a certain acoustic event would be observed in 95 out of 100 different experiments.

For two sinusoidal signals of the same frequency and specified phase lag (in the absence of noise), the cross-spectral phase at the signal frequency is equal to the phase lag between the signals. When dealing with real data, however, noise is superimposed causing reduced coherency and cross-spectral phase errors. Additionally, some form of averaging must be performed on the cross-spectra in order to obtain a coherency spectrum (Jenkins and Watts, 1968). This significantly increases the bandwidth of the cross-spectra, with the result that cross-spectral phases are biased by noise and other signals within the 
bandwidth of the cross-spectra.

In light of the above, we estimate the reliability of cross-spectral phases using the barotropic tidal signals in the tomographic data. Headrick (1990) has analyzed the barotropic tidal signals in the tomographic data, and finds close agreement between the data and models of the diurnal tides. A continuous 110-day data segment is available from four receivers. Dividing these data sets into consecutive 55-day sections, we compute cross-spectra for each receiver combination using the same processing and averaging used on the 42-day data segments discussed in the body of this paper. At the diurnal tide, $\mathrm{K}_{1}$, cross-spectral phases obtained from each 55-day segment at each receiver combination is consistent within $\pm 15^{\circ}$. We therefore use $\pm 15^{\circ}$ as a measure of the reliability of our cross-spectral phases. 


\section{Appendix C}

\section{A Two-Mode Gravity Wave}

\section{Model}

Let the observed acoustic travel-time at receiver $i$ be,

$$
c_{i} \sin \left(\sigma t+\theta_{i}\right)
$$

where $c_{i}$ is the magnitude of the rms travel-time estimated from auto-spectra, $\theta_{i}$ is the cross-spectral phase (referenced to R3), $\sigma$ is the mode frequency, and $t$ is time. We model the acoustic travel-time at receiver $i$ from a linear combination of the clockwise and counterclockwise travelling resonant barotropic gravity waves (Section 4.4.1) as,

$$
\tilde{c}_{i} \sin \left(\sigma+\tilde{\theta}_{i}\right)=\alpha_{1} a_{i 1} \sin \left(\sigma+\phi_{i 1}+\zeta_{1}\right)+\alpha_{2} a_{i 2} \sin \left(-\sigma+\phi_{i 2}+\zeta_{2}\right)
$$

where the variables with tildes are the model estimates, and the right side of the equation corresponds to the summation of the clockwise and counterclockwise modes.

Thus for each mode we have four model parameters, $\left(\alpha_{1}, \alpha_{2}, \zeta_{1}, \zeta_{2}\right)$, which are varied to minimize the error between the observed travel-times and the model estimates. We 
establish a relevant (but somewhat arbitrary) cost function, $J$, which evaluates how well the two-mode model fits the observed data at all receivers for a given mode. After some trial and error, we selected a cost function which penalizes percentage errors in magnitude and deviations in phase between the model and the data. The cost function selected is,

$$
J\left(\alpha_{1}, \alpha_{2}, \zeta_{1}, \zeta_{2}\right)=\sum_{i=1}^{7}\left(\frac{c_{i}}{\tilde{c}_{i}}+\frac{\tilde{c}_{i}}{c_{i}}\right)^{4}+100 \cdot\left(\tilde{\theta}_{i}-\theta_{i}\right)^{2},
$$

where the summation is taken over all receivers.

For each mode we find, after minimizing the cost function, that the magnitude and phase obtained from the two-mode model of resonant barotropic gravity waves performed no better than the results using a single mode model. 


\section{Bibliography}

Blanding, W.R., J. Spiesberger, and A. Miller, Observations of ocean fluctuations between 15 and 23 hour periods in the Pacific, J. Geo. Res., (in preparation), 1990.

DeFerrari, H.A. and G.B. Nguyen, Acoustic reciprocal transmission experiment, Florida Straits, J. Acoust. Soc. Am., 79, 299-315, 1986.

Ewing, M. and J.L. Worzel, Long-range sound transmission, Geol. Soc. Am. Memo, 27, 1948.

Harris, F.J., On the use of windows for harmonic analysis with the discrete Fourier transform, Proc. IEEE, 66, 51-83, 1978.

Headrick, R.H., Basin-scale tidal measurements using acoustic tomography, Master's thesis, Woods Whole Oceanographic Institute-Massachusetts Institute of Technology, 45pp, 1990 .

Jenkins, G.M. and D.G. Watts, Spectral Analysis and Its Applications, 525pp, HoldenDay, San Francisco, 1968.

Longuet-Higgins, M.S., On the trapping of wave energy round islands, J. Fluid Mech., 29, 781-821, 1967.

Luther, D.S., Trapped waves around the Hawaiian Islands, Proceedings, 'Aha Huliko'a Hawaiian Winter Workshop, Univ. of Hawaii, January 21-23, 1985, 261-301.

Metzger, K., Signal processing equipment and techniques for use in measuring ocean acoustic multipath structures, Ph.D. thesis, Univ. of Michigan, Ann Arbor, 1983.

Miller, A., W.R. Holland, and M.C. Hendershott, Open-ocean response and normal mode excitation in an eddy-resolving general circulation model, Geophys. Astrophys. Fluid Dyn., 37, 253-278, 1988.

Munk, W., and C. Wunsch, Ocean acoustic tomography: A scheme for large scale monitoring, Deep Sea Res., 26, 123-161, 1979.

Officer, C.B., Introduction to the Theory of Sound Transmission, McGraw-Hill, New York, 1958 .

Patzert, W.C. and K. Wyrtki, Anticyclonic flow around the Hawaiian Islands indicated by current meter data, J. Phys. Oceanogr., 4, 673-676, 1974.

Platzman, G.W., Normal modes of the world ocean. Part I. Design of a finite-element barotropic mode, J. Phys. Oceanogr., 8, 1073- 1090, 1978. 
Platzman, G.W., G.A. Curtis, K.S. Hansen, and R.D. Slater, Normal modes of the world ocean. Part II: description of modes in the period range 8 to 80 hours, J. Phys. Oceanogr., 11, 579-603, 1981.

Proudman, J., Diffraction of tidal waves on flat rotating sheets of water, Proc. Lond. Math Soc., 14-2, 89-102, 1914.

Spiesberger, J.L., T.G. Birdsall, K. Metzger, R.A. Knox, C.W. Spofford, and R.C. Spindel, Measurements of Gulf Stream meandering and evidence of seasonal thermocline development using long- range acoustic transmissions, J. Phys. Oceanogr., 13, 1836-1846, 1983.

Spiesberger, J.L., P.J. Bushong, K. Metzger, and T.G. Birdsall, Ocean acoustic tomography: Estimating the acoustic travel time with phase, IEEE J. Oceanogr. Eng., 14, 108-119, $1989 a$.

Spiesberger, J.L., P.J. Bushong, K. Metzger, and T.G. Birdsall, Basin-scale tomography: Synoptic measurements of a 4000-km length section in the Pacific, J. Phys. Oceanogr., 19, 1073-1090, $1989 b$.

Summerfield. W., Circular islands as resonators of long-wave energy, Phil. Trans. R. Soc. Lond., A272, 361-402, 1972.

Urick, R.J., Principles of Underwater Sound, 423pp, McGraw-Hill, New York, 1983.

Wunsch, C., The spectrum from two years to two minutes of temperature fluctuations in the main thermocline at Bermuda, Deep Sea Res., 19, 577-593, 1972. 